\title{
Hopf Modules in the Category of Yetter-Drinfeld Modules
}

\author{
Yanmin Yin \\ Department of Mathematics, Shandong Jianzhu University, Jinan, China \\ Email:yanmin_yin@163.com \\ Received 8 August 2015; accepted 24 April 2016; published 27 April 2016 \\ Copyright (C) 2016 by author and Scientific Research Publishing Inc. \\ This work is licensed under the Creative Commons Attribution International License (CC BY). \\ http://creativecommons.org/licenses/by/4.0/ \\ (c) (i) Open Access
}

\begin{abstract}
We give the Fundamental Theorem for Hopf modules in the category of Yetter-Drinfeld modules ${ }_{L}^{L} \mathscr{Q} \mathscr{Q}$, where $L$ is a quasitriangular weak Hopf algebra with a bijective antipode. We also show that $\boldsymbol{H}^{*}$ has a right $\boldsymbol{H}$-Hopf module structure in the Yetter-Drinfeld category. As an application we deduce the existence and uniqueness of right integral from it.
\end{abstract}

Keywords

Weak Hopf Algebra, Hopf Module, Fundamental Theorem

\section{Introduction}

Weak Hopf algebras were introduced by G. Böhm and K. Szlachányi as a generalization of usual Hopf algebras and groupoid algebras [1] [2]. A weak Hopf algebra is a vector space that has both algebra and coalgebra structures related to each other in a certain self-dual fashion and possesses an analogue of the linearized inverse map [3]-[5]. The main difference between ordinary and weak Hopf algebras comes from the fact that the comultiplication of the latter is no longer required to preserve the unit (equivalently, the counit is not requires to be a homomorphism) and results in the existence of two canonical subalgebras playing the role of "noncommutative bases".

Paper [6] was shown what is a weak Hopf algebra in the braided category of modules over a weak Hopf algebra. In [7] we prove a Fundamental Theorem of Hopf modules for the categorical weak Hopf algebra motivation to study quasitriangular weak Hopf algebras is the so-called biproduct construction and interpreted in the terms of braided categories. More precisely, we are interested in a specific type of quaitriangular weak Hopf algebras.

we prove the Fundamental Theorem for Hopf modules in the category of Yetter-Drinfeld modules according to the fact that the matrix $R$ gives rise to a natural braiding for ${ }_{L} / /$ and ${ }_{L}^{L} g /$. Furthermore $H^{*}$ is also a right $H$-Hopf module in the category Yetter-Drinfeld modules. Using this result we obtain the existence and 
uniqueness of integrals for a finite dimensional weak Hopf algebra in ${ }_{L}^{L} Y$.

\section{Preliminaries}

Throughout this paper we use Sweedler's notation for comultiplication, writing $\Delta(h)=h_{1} \otimes h_{2}$. Let $k$ be a fixed field and all weak Hopf algebras are finite dimensional.

Definition 1. A weak Hopf algebra is a vector space $L$ with the structure of an associative unital algebra $(L, m, \mu)$ with multiplication $m: L \otimes L \rightarrow L$ and unit $1 \in L$ and a coassociative coalgebra $(L, \Delta, \varepsilon)$ with comultiplication $\Delta: L \rightarrow L \otimes L$ and counit $\varepsilon: L \rightarrow k$ such that

1) The comultiplication $\Delta$ is a (not necessarily unit-preserving) homomorphism of algebras such that

$$
(\Delta \otimes \text { id }) \Delta(1)=(\Delta(1) \otimes 1)(1 \otimes \Delta(1))=(1 \otimes \Delta(1))(\Delta(1) \otimes 1) .
$$

2) The counit satisfies the following identity

$$
\varepsilon(k g l)=\varepsilon\left(k g_{1}\right) \varepsilon\left(g_{2} l\right)=\varepsilon\left(k g_{2}\right) \varepsilon\left(g_{1} l\right), \quad \forall k, g, l \in L .
$$

3) There is a linear map $S_{L}: L \rightarrow L$ called an antipode, such that, for all $l \in L$

$$
\begin{gathered}
m\left(i d \otimes S_{L}\right) \Delta(l)=(\varepsilon \otimes i d)(\Delta(1)(l \otimes 1)), \\
m\left(S_{L} \otimes i d\right) \Delta(l)=(i d \otimes \varepsilon)((1 \otimes l) \Delta(1)), \\
S_{L}(l)=S_{L}\left(l_{1}\right) l_{2} S_{L}\left(l_{3}\right) .
\end{gathered}
$$

The linear map defined in the above equations are called target and source counital maps and denoted by $\varepsilon_{t}$ and $\varepsilon_{s}$ respectively:

$$
\begin{aligned}
& \varepsilon_{t}(l)=\varepsilon\left(1_{(1)} l\right) 1_{(2)}=\varepsilon\left(S_{L}(l) 1_{(1)}\right) 1_{(2)}, \\
& \varepsilon_{s}(l)=1_{(1)} \varepsilon\left(l 1_{(2)}\right)=1_{(1)} \varepsilon\left(1_{(2)} S_{L}(l)\right) .
\end{aligned}
$$

For all $l \in L$, we have

$$
\begin{gathered}
l_{1} \otimes \varepsilon_{t}\left(l_{2}\right)=1_{(1)} l \otimes 1_{(2)}, \quad \varepsilon_{s}\left(l_{1}\right) \otimes l_{2}=1_{(1)} \otimes l 1_{(2)}, \\
l_{1} \otimes \varepsilon_{s}\left(l_{2}\right)=l 1_{(1)} \otimes S_{L}\left(1_{(2)}\right), \quad \varepsilon_{t}\left(l_{1}\right) \otimes l_{2}=S_{L}\left(1_{(1)}\right) \otimes 1_{(2)} l .
\end{gathered}
$$

We will briefly recall the necessary definitions and notions on the weak Hopf algebras.

Definition 2. A quasitriangular weak Hopf algebra is a pair $(L, R)$ where $L$ is a weak Hopf algebra and $R \in \Delta^{o p}(1)(L \otimes L) \Delta(1)$ (called the $R$-matrix) satisfying the following conditions:

$$
\Delta^{o p}(l) R=R \Delta(l)
$$

for all $l \in L$, where $\Delta^{o p}$ denotes the conditions apposite to $\Delta$,

$$
\begin{aligned}
& (\text { id } \otimes \Delta)(R)=R_{13} R_{12}, \\
& (\Delta \otimes i d)(R)=R_{13} R_{23} .
\end{aligned}
$$

where $R_{12}=R \otimes 1, R_{23}=1 \otimes R$, etc. as usual, and such that there exits $\bar{R} \in \Delta(1)(L \otimes L) \Delta^{\text {op }}(1)$ with $R \bar{R}=\Delta^{o p}(1), \bar{R} R=\Delta(1)$. where we write $R=R^{1} \otimes R^{2}=r^{1} \otimes r^{2}=T^{1} \otimes T^{2}$. By [3], we can obtain the following results.

Proposition 2.1. For any quasitriangular weak Hopf algebra $(L, R)$, we have

$$
\begin{aligned}
& \left(\varepsilon_{s} \otimes i d\right)(R)=\Delta(1), \quad\left(i d \otimes \varepsilon_{s}\right)(R)=\left(S_{L}^{-1} \otimes i d\right) \Delta^{o p}(1), \\
& \left(\varepsilon_{t} \otimes i d\right)(R)=\Delta^{o p}(1), \quad\left(i d \otimes \varepsilon_{t}\right)(R)=\left(S_{L}^{-1} \otimes i d\right) \Delta(1), \\
& \left(S_{L} \otimes i d\right)(R)=\left(i d \otimes S_{L}^{-1}\right)(R)=\bar{R}, \\
& \left(S_{L} \otimes S_{L}\right)(R)=R, \quad(\varepsilon \otimes i d)(R)=(i d \otimes R)=1 .
\end{aligned}
$$




\section{Weak Hopf Algebras in the Yetter-Drinfeld Module Category}

Let $L$ be a quasitriangular weak Hopf algebra with a bijective antipode $S_{L}$. Suppose $H$ is a weak Hopf algebra in ${ }_{L} . / /$. Paper [7] show that $H$ is also a weak Hopf algebra in ${ }_{L}^{L} g Y$ with a left $L$-coaction via

$\sigma_{H}: H \rightarrow L \otimes H, h \mapsto h^{-1} \otimes h^{0}=R^{2} \otimes R^{1} \rightarrow h$. Bing-liang and Shuan-hong introduce the definition of Weak Hopf algebra in the braided monoidal category ${ }_{L}^{L} \mathscr{C}$ in [6]. Moreover they have showed that if $H$ is a finite-dimensional weak Hopf algebra in ${ }_{L}^{L} \mathscr{Y O}$, then its dual $H^{*}$ is a weak Hopf algebra in ${ }_{L}^{L} \mathscr{Y O}$.

Definition 3. Let $(L, R)$ be a quasitriangular weak Hopf algebra. An object $H \in{ }_{L}^{L} \mathscr{Y} g$ is called a weak bialgebra in this category if it is both an algebra and a coalgebra satisfying the following conditions:

1) $\Delta$ and $\varepsilon$ are not necessarily unit-preserving, such that

$$
\begin{aligned}
& \Delta(x y)=x_{1}\left(R^{2} \rightarrow y_{1}\right) \otimes\left(R^{1} \rightarrow x_{2}\right) y_{2}, \\
& \varepsilon(x y z)=\varepsilon\left(x y_{1}\right) \varepsilon\left(y_{2} z\right)=\varepsilon\left(x\left(R^{2} \rightarrow y_{2}\right)\right) \varepsilon\left(\left(R^{1} \rightarrow y_{1}\right) z\right), \\
& \Delta^{2}(1)=1_{1} \otimes 1_{2} 1_{1}^{\prime} \otimes 1_{2}^{\prime}=1_{1} \otimes\left(R^{2} \rightarrow 1_{1}^{\prime}\right)\left(R^{1} \rightarrow 1_{2}\right) \otimes 1_{2}^{\prime} .
\end{aligned}
$$

2) $H$ is a left $L$-module algebra and left $L$-module coalgebra if $H$ is a left $L$-module via $l \otimes x \mapsto l \rightarrow x$ such that

$$
\begin{gathered}
l \rightarrow x y=\left(l_{1} \rightarrow x\right)\left(l_{2} \rightarrow y\right), l \rightarrow 1=\varepsilon_{t}(l) \rightarrow 1, x, y \in H, l \in L, \\
\Delta(l \rightarrow x)=\left(l_{1} \rightarrow x_{1}\right) \otimes\left(l_{2} \rightarrow x_{2}\right), \varepsilon_{s}(l) \rightarrow x=x_{1} \varepsilon\left(l \rightarrow x_{2}\right) .
\end{gathered}
$$

3) $H$ is a left $L$-comodule algebra and left $L$-comodule coalgebra if $H$ is a left $L$-comodule via $x \mapsto \sigma_{H}(x)=x^{-1} \otimes x^{0}=R^{2} \otimes R^{1} \rightarrow x$ such that

$$
\begin{aligned}
& \sigma_{H}(x y)=x^{-1} y^{-1} \otimes x^{0} y^{0}=R^{2} r^{2} \otimes\left(R^{1} \rightarrow x\right)\left(r^{1} \rightarrow y\right), \\
& R^{2} \otimes x\left(R^{1} \rightarrow 1\right)=\varepsilon_{s}\left(R^{2}\right) \otimes\left(R^{1} \rightarrow x\right), \\
& R^{2} \otimes\left(R^{1} \rightarrow x\right)_{1} \otimes\left(R^{1} \rightarrow x\right)_{2}=R^{2} r^{2} \otimes R^{1} \rightarrow x_{1} \otimes r^{1} \rightarrow x_{2}, \\
& \varepsilon\left(R^{1} \rightarrow x\right) R^{2}=\varepsilon\left(R^{1} \rightarrow x\right) \varepsilon_{t}\left(R^{2}\right) .
\end{aligned}
$$

4) Furthermore, $H$ is called a weak Hopf algebra in ${ }_{L}^{l} g O$ if there exists an antipode $S: H \rightarrow H$ (here $S$ is left $L$-linear and left $L$-colinear i.e., $S$ is a morphism in the category of ${ }_{L}^{L} \mathscr{Y O}$ ) satisfying

$$
\begin{aligned}
& x_{1} S\left(x_{2}\right)=\varepsilon\left(\left(R^{2} \rightarrow 1_{1}\right)\left(R^{1} \rightarrow x\right)\right) 1_{2}, \\
& S\left(x_{1}\right) x_{2}=1_{1} \varepsilon\left(\left(R^{2} \rightarrow x\right)\left(R^{1} \rightarrow 1_{2}\right)\right), \\
& S\left(x_{1}\right) x_{2} S\left(x_{3}\right)=S(x), \forall x \in H .
\end{aligned}
$$

Similar to the definition of weak Hopf algebra, we denote $\varepsilon_{t}(x)=x_{1} S\left(x_{2}\right), \varepsilon_{s}(x)=S\left(x_{1}\right) x_{2}$. If $x=1$ one can obtain $\varepsilon_{t}(1)=\varepsilon_{s}(1)=1$. According to the definitions of $\varepsilon_{t}, \varepsilon_{s}$ one obtains explicit expressions for these coproducts

$$
\Delta\left(\varepsilon_{t}(x)\right)=\varepsilon_{t}(x) 1_{1} \otimes 1_{2}, \quad \Delta(\varepsilon(x))=1_{1} \otimes 1_{2} \varepsilon_{s}(x) .
$$

Paper [7] give the following results:

Proposition 3.1. Suppose $H$ is a weak Hopf algebra in ${ }_{L}^{L} \mathscr{Q}$. For all $x \in H$ we have the identities

$$
x_{1} \otimes \varepsilon_{s}\left(x_{2}\right)=x 1_{1} \otimes S\left(1_{2}\right), \quad \varepsilon_{t}\left(x_{1}\right) \otimes x_{2}=S\left(1_{1}\right) \otimes 1_{2} x .
$$

Since a weak Hopf algebra $H$ in the weak Yetter-Drinfeld categories ${ }_{L}^{L} \mathscr{P}$ is both algebra and coalgebra, one can consider modules and comodules over $H$. As in the theory of Hopf algebras, an H-Hopf module is an $H$-module which is also an $H$-comodule such that these two structures are compatible (the action "commutes" with coaction):

Definition 4. Let $H$ be a weak Hopf algebra in ${ }_{L}^{L} \mathscr{G}$. A right $H$-Hopf module $M$ in ${ }_{L}^{L} g(Y)$ is an object $M \in{ }_{L}^{L} \mathscr{Q}$ such that it is both a right $H$-module $\varphi_{M}: M \otimes H \rightarrow M$ and a right $H$-comodule via 
$\rho_{M}: M \rightarrow M \otimes H, \rho_{M}(m)=m_{0} \otimes m_{1}$ and the following equations hold for $m \in M, h \in H, l \in L:$

1) $\rho_{M}(m h)=m_{0}\left(R^{2} \rightarrow h_{1}\right) \otimes\left(R^{1} \rightarrow m_{1}\right) h_{2}$,

2) $l \rightarrow(m h)=\left(l_{1} \rightarrow m\right)\left(l_{2} \rightarrow h\right)$,

3) $R^{2} \otimes\left(R^{1} \rightarrow m\right)_{0} \otimes\left(R^{1} \rightarrow m\right)_{1}=R^{2} r^{2} \otimes\left(R^{1} \rightarrow m_{0}\right) \otimes\left(r^{1} \rightarrow m_{1}\right)$,

4) $\sigma_{M}(m h)=R^{2} r^{2} \otimes\left(R^{1} \rightarrow m\right)\left(r^{1} \rightarrow h\right)$,

5) $\rho_{M}(l \rightarrow m)=\left(l_{1} \rightarrow m_{0}\right)\left(l_{2} \rightarrow m_{1}\right)$.

We remark that $M \otimes_{t} H$ is a right $H$-module by $(m \otimes h) x=m\left(R^{2} \rightarrow x_{1}\right) \otimes\left(R^{1} \rightarrow h\right) x_{2}$ and a right $H$-comodule $\rho_{M \otimes H}(m \otimes h)=m_{0} \otimes R^{2} \rightarrow h_{1} \otimes\left(R^{1} \rightarrow m_{1}\right) h_{2}$. The condition (1) means that the $H$-comodule structure $\rho_{M}: M \rightarrow M \otimes H$ is $H$-linear, or equivalently the $H$-module structure map $\varphi_{M}: M \otimes H \rightarrow M$ is $H$ colinear. Also, (4) (resp. (2)) $\Leftrightarrow \varphi_{M}$ is $L$-colinear (resp. $L$-linear); (3)(resp. (5)) $\Leftrightarrow \rho_{M}$ is $L$-colinear (resp. $L$-linear).

Example 3.2. $H$ itself is a right $H$-Hopf module (in ${ }_{L}^{L} \mathscr{Y O}$ ) in the natural way. If $V$ is an object in ${ }_{L}^{L} \mathscr{Y O}$, then so is $V \otimes_{t} H$ by $l \rightarrow(v \otimes h)=\left(l_{1} \rightarrow v\right) \otimes\left(l_{2} \rightarrow h\right)$ and $\sigma_{v \otimes H}(v \otimes h)=R^{2} r^{2} \otimes\left(R^{1} \rightarrow v\right) \otimes\left(r^{1} \rightarrow h\right)$. It is also both a right $H$-module and a right $H$-comodule by $(v \otimes h) x=v \otimes h x$ and $\rho_{V \otimes H}(v \otimes h)=v \otimes h_{1} \otimes h_{2}$. One easily checks that $V \otimes_{t} H$ is an right $H$-Hopf module.

when $H$ is a weak Hopf algebra in ${ }_{L} / /$ and $M$ a right $H$-Hopf module in ${ }_{L} / /$, we prove the Fundamental Theorem 3.3 [7]. Furthermore we will show $M^{c o H}=\left\{m \in M \mid \rho_{M}(m)=m 1_{1} \otimes 1_{2}\right\}$ is a $L$-subcomodule of $M$.

Applying $R^{2} \otimes R^{1} \rightarrow 1=R^{2} \otimes \varepsilon_{t}\left(R^{1}\right) \rightarrow 1=1_{(1)} \otimes\left(1_{(2)} \rightarrow 1\right)$ we obtain

$$
\begin{aligned}
R^{2} \otimes\left(R^{1} \rightarrow 1\right)_{1} \otimes\left(R^{1} \rightarrow 1\right)_{2} & =1_{(1)} \otimes\left(1_{(2)} \rightarrow 1_{1}\right) \otimes 1_{2} \\
& =1_{(1)} \otimes\left(\varepsilon_{t}\left(1_{(2)}\right) \rightarrow 1_{1}\right) \otimes 1_{2} \\
& =1_{(1)} \otimes\left(1_{(2)} \rightarrow 1\right) 1_{1} \otimes 1_{2} \\
& =R^{2} \otimes\left(R^{1} \rightarrow 1\right) 1_{1} \otimes 1_{2} .
\end{aligned}
$$

For $n \in M^{\mathrm{coH}}$ we do a calculation:

$$
\begin{aligned}
n^{-1} \otimes\left(n^{0}\right)_{1} \otimes\left(n^{0}\right)_{2} & =R^{2} \otimes\left(R^{1} \rightarrow n\right)_{0} \otimes\left(R^{1} \rightarrow n\right)_{1} \\
& =R^{2} r^{2} \otimes\left(R^{1} \rightarrow n 1_{1}\right) \otimes\left(r^{1} \rightarrow 1_{2}\right) \\
& =R^{2} T^{2} r^{2} \otimes\left(R^{1} \rightarrow n\right)\left(T^{1} \rightarrow 1_{1}\right) \otimes\left(r^{1} \rightarrow 1_{2}\right) \\
& =R^{2} r^{2} \otimes\left(R^{1} \rightarrow n\right)\left(r^{1} \rightarrow 1\right)_{1} \otimes\left(r^{1} \rightarrow 1\right)_{2} \\
& =R^{2} r^{2} \otimes\left(R^{1} \rightarrow n\right)\left(r^{1} \rightarrow 1\right) 1_{1} \otimes 1_{2} \\
& =R^{2} \otimes\left(R^{1} \rightarrow n\right) 1_{1} \otimes 1_{2} \\
& =n^{-1} \otimes n^{0} 1_{1} \otimes 1_{2} .
\end{aligned}
$$

This implies that $\sigma(n)=n^{-1} \otimes n^{0} \in L \otimes M^{c o H}$. So $M^{c o H} \in{ }_{L}^{L} Y O$.

It is clearly to prove $F$ is a left $L$-colinear by the following equation

$$
\sigma(F(n \otimes h))=\sigma(n h)=n 1_{(1)} R^{2} \otimes 1_{(2)}\left(R^{1} \rightarrow h\right)=(i d \otimes F) \sigma(n \otimes h) .
$$

Furthermore we can obtain the Structure Theorem for right $H$-Hopf modules in the category of YetterDrinfeld modules.

Theorem 3.3. If $H$ is a weak Hopf algebra in ${ }_{L}^{L} Y Q$ and $M$ is a right $H$-Hopf module in ${ }_{L}^{L} \mathscr{Y}, M^{\text {coH }}$ is defined as above. Then

1) Let $P(m)=m_{0} S\left(m_{1}\right), m \in M$. Then $P(m) \in M^{c o H}$. If $n \in M^{c o H}$ and $h \in H$, Then $\rho_{M}(n h)=n h_{1} \otimes h_{2}$ and $P(n h)=n \varepsilon_{t}(h)$.

2) The map $F: M^{c o H} \otimes_{t} H \rightarrow M, F(n \otimes h)=n h$ is an isomorphism of Hopf modules. The inverse map is 
given by $G(m)=P\left(m_{0}\right) m_{1}$.

\section{Fundamental Theorem for $H^{*}$ in ${ }_{L}^{L} \mathscr{Y Q O}$}

In [4] $H^{*}$ has the contragredient left $L$-module structure by

$$
(l \rightarrow f)(h)=f\left(S_{L}(l) \rightarrow h\right), \quad l \in L, f \in H^{*}, h \in H .
$$

Since $H$ is a finite-dimensional left $L$-comodule, $H^{*}$ has the transposed right $L$-comodule structure and so it becomes a left $L$-comodule via

$$
\sigma_{H^{*}}: H^{*} \rightarrow L \otimes H^{*}, \sigma_{H^{*}}(f)=f^{-1} \otimes f^{0}=R^{2} \otimes R^{1} \rightarrow f .
$$

i.e. $f^{0}(h) f^{-1}=f\left(h^{0}\right) S_{L}^{-1}\left(h^{-1}\right)=f\left(R^{1} \rightarrow h\right) S^{-1}\left(R^{2}\right), h \in H$. Now assume that $H$ is finite-dimensional. We will show that $H^{*}$ becomes a right $H$-Hopf module in ${ }_{L}^{L} g\left(\right.$. First $H^{*}$ is a right $H$-module by

$$
(f h)(x)=f(h x), f \in H^{*}, h \in H .
$$

Second, $H^{*}$ is a right $H$-comodule using the identification $\theta_{H}: H^{*} \otimes H \cong H o m(H, H)$, $\theta_{H}(f \otimes h)(x)=f\left(R^{2} \rightarrow x\right)\left(R^{1} \rightarrow h\right)$ as follows:

$$
\rho_{H^{*}}: H^{*} \rightarrow \operatorname{Hom}(H, H) \cong H^{*} \otimes H, \rho_{H^{*}}(f)(x)=f\left(x_{1}\right) S\left(x_{2}\right) .
$$

That is $\rho_{H^{*}}(f)=f_{0} \otimes f_{1}$ means

$$
f\left(x_{1}\right) S\left(x_{2}\right)=f_{0}\left(f_{1}^{-1} \rightarrow x\right) f_{1}^{0}=f_{0}\left(R^{2} \rightarrow x\right)\left(R^{1} \rightarrow f_{1}\right), \quad x \in H .
$$

Proposition 4.1. $H^{*}$ is a right $H$-comodule by $\theta_{H \otimes H}$.

Proof. Now for $f \in H^{*}, x \in H$, we have

$$
\begin{aligned}
& \theta_{H \otimes H}\left(\left(f_{0}\right)_{0} \otimes\left(f_{0}\right)_{1} \otimes f_{1}\right)(x) \\
& =\left(f_{0}\right)_{0}\left(r^{2} \rightarrow\left(R^{2} \rightarrow x\right)\right)\left(r^{1} \rightarrow\left(f_{0}\right)_{1}\right) \otimes R^{1} \rightarrow f_{1}, \\
& =f_{0}\left(\left(R^{2}\right)_{1} \rightarrow x_{1}\right) S\left(\left(R^{2}\right)_{2} \rightarrow x_{2}\right) \otimes R^{1} \rightarrow f_{1}, \\
& =f\left(x_{1}\right)\left(R^{2} \rightarrow S\left(x_{3}\right)\right) \otimes R^{1} \rightarrow S\left(x_{2}\right), \\
& =\Delta_{H}\left(f\left(x_{1}\right) S\left(x_{2}\right)\right), \\
& =f_{0}\left(R^{2} \rightarrow x\right)\left(\left(R^{1}\right)_{1} \rightarrow\left(f_{1}\right)_{1}\right) \otimes\left(\left(R^{1}\right)_{2} \rightarrow\left(f_{1}\right)_{2}\right), \\
& =f_{0}\left(R^{2} r^{2} \rightarrow x\right)\left(R^{1} \rightarrow\left(f_{1}\right)_{1}\right) \otimes\left(r^{1} \rightarrow\left(f_{1}\right)_{2}\right), \\
& =\theta_{H \otimes H}\left(f_{0} \otimes\left(f_{1}\right)_{1} \otimes\left(f_{1}\right)_{2}\right)(x) .
\end{aligned}
$$

It implies that $\left(f_{0}\right)_{0} \otimes\left(f_{0}\right)_{1} \otimes f_{1}=f_{0} \otimes\left(f_{1}\right)_{1} \otimes\left(f_{1}\right)_{2}$.

Accord to $\rho_{H^{*}}(f) \in{ }_{L}^{L} \mathscr{Y O}$ we have $\left(1_{(1)} \rightarrow f_{0}\right) \otimes\left(1_{(2)} \rightarrow f_{1}\right)=f_{0} \otimes f_{1}$. Applying the equality $\varepsilon\left(R^{1} \rightarrow x\right) R^{2}=\varepsilon\left(R^{1} \rightarrow x\right) \varepsilon_{t}\left(R^{2}\right)$ we obtain

$$
\begin{aligned}
f(x) & =f_{0}\left(R^{2} \rightarrow x\right) \varepsilon\left(R^{1} \rightarrow f_{1}\right) \\
& =f_{0}\left(\varepsilon_{t}\left(R^{2}\right) \rightarrow x\right) \varepsilon\left(R^{1} \rightarrow f_{1}\right) \\
& =f_{0}\left(S_{L}\left(1_{(1)}\right) \rightarrow x\right) \varepsilon\left(1_{(2)} \rightarrow f_{1}\right) \\
& =\left(1_{(1)} \rightarrow f_{0}\right)(x) \varepsilon\left(1_{(2)} \rightarrow f_{1}\right), \\
& =\left(f_{0} \varepsilon\left(f_{1}\right)\right)(x) .
\end{aligned}
$$

Hence $($ id $\otimes \varepsilon) \rho_{H^{*}}(f)=f$. Thus $H^{*}$ becomes a right $H$-comodule. 
Theorem 4.2. With the notation as above, then $H^{*}$ is a right $H$-Hopf module in ${ }_{L}^{L} \mathscr{Y O}$. Moreover,

$$
\left(H^{*}\right)^{c o H}=\left\{f \in H^{*} \mid f\left(x_{1}\right) S\left(x_{2}\right)=f\left(1_{1} x\right) 1_{2}, x \in H\right\} .
$$

Proof. Now we prove that $H^{*}$ is a right $H$-Hopf module. First we will show that $\rho(f h)=f_{0}\left(R^{2} \rightarrow h_{1}\right) \otimes\left(R^{1} \rightarrow f_{1}\right) h_{2}$. Since for $x \in H$,

$$
\begin{aligned}
& \theta_{H}\left(f_{0}\left(R^{2} \rightarrow h_{1}\right) \otimes\left(R^{1} \rightarrow f_{1}\right) h_{2}\right)(x) \\
& =f_{0}\left(\left(R^{2} \rightarrow h_{1}\right)\left(r^{2} T^{2} \rightarrow x\right)\right)\left(r^{1} R^{1} \rightarrow f_{1}\right)\left(T^{1} \rightarrow h_{2}\right) \\
& =f_{0}\left(R^{2} \rightarrow h_{1}\left(T^{2} \rightarrow x\right)\right)\left(R^{1} \rightarrow f_{1}\right)\left(T^{1} \rightarrow h_{2}\right) \\
& =f\left(\left(h_{1}\left(T^{2} \rightarrow x\right)\right)_{1}\right) S\left(\left(h_{1}\left(T^{2} \rightarrow x\right)\right)_{2}\right)\left(T^{1} \rightarrow h_{2}\right) \\
& =f\left(h_{1}\left(\left(R^{2}\right)_{1} \rightarrow x_{1}\right)\right)\left(\left(R^{2}\right)_{2} \rightarrow S\left(x_{2}\right)\right)\left(R^{1} \rightarrow \varepsilon_{s}\left(h_{2}\right)\right) \\
& =f\left(h 1_{1}\left(R^{2} \rightarrow x_{1}\right)\right)\left(r^{2} \rightarrow S\left(x_{2}\right)\right)\left(r^{1} \rightarrow\left(R^{1} \rightarrow S\left(1_{2}\right)\right)\right) \\
& =(f \cdot h)\left(1_{1}\left(R^{2} \rightarrow x_{1}\right)\right) S\left(\left(R^{1} \rightarrow 1_{2}\right) x_{2}\right)=\left[(f \cdot h)_{0}\right] \otimes\left[(f \cdot h)_{1}\right](x) .
\end{aligned}
$$

Next we want to check $(l \rightarrow f h)=\left(l_{1} \rightarrow f\right)\left(l_{2} \rightarrow h\right)$ for $l \in L, h \in H, f \in H^{*}$. Since for $x \in H$

$$
\begin{aligned}
& \left(\left(l_{1} \rightarrow f\right)\left(l_{2} \rightarrow h\right)\right)(x) \\
= & \left(l_{1} \rightarrow f\right)\left(\left(l_{2} \rightarrow h\right) x\right) \\
= & f\left(S_{L}\left(l_{1}\right) \rightarrow\left(\left(l_{2} \rightarrow h\right) x\right)\right) \\
= & f\left(\left(S_{L}\left(l_{2}\right) l_{3} \rightarrow h\right)\left(S_{L}\left(l_{1}\right) \rightarrow x\right)\right) \\
= & f\left(\left(\varepsilon_{s}\left(l_{2}\right) \rightarrow h\right)\left(S_{L}\left(l_{1}\right) \rightarrow x\right)\right) \\
= & f\left(\left(S_{L}\left(1_{(2)}\right) \rightarrow h\right)\left(S_{L}\left(l_{(1)}\right) \rightarrow x\right)\right) \\
= & f\left(\left(1_{(1)} \rightarrow h\right)\left(1_{(2)} \rightarrow\left(S_{L}(l) \rightarrow x\right)\right)\right) \\
= & (f h)\left(S_{L}(l) \rightarrow x\right)=(l \rightarrow f h)(x) .
\end{aligned}
$$

Applying the equality $R^{1} S\left(l_{2}\right) \otimes S\left(l_{1}\right) R^{2}=l_{1} R^{1} \otimes R^{2} S\left(l_{2}\right)$ for $x \in H$

$$
\begin{aligned}
& R^{2} r^{2} \otimes \theta_{H}\left(\left(R^{1} \rightarrow f_{0}\right) \otimes\left(r^{1} \rightarrow f_{1}\right)\right)(x) \\
& =R^{2} f_{0}\left(S\left(\left(R^{1}\right)_{1}\right) r^{2} \rightarrow x\right)\left(r^{1}\left(\left(R^{1}\right)_{2}\right) \rightarrow f_{1}\right) \\
& =R^{2} f_{0}\left(r^{2} \rightarrow\left(S\left(\left(R^{1}\right)_{2}\right) \rightarrow x\right)\right)\left(\left(R^{1}\right)_{1} r^{1} \rightarrow f_{1}\right) \\
& =R^{2} f\left(\left(S\left(\left(R^{1}\right)_{2}\right) \rightarrow x\right)_{1}\right)\left(\left(R^{1}\right)_{1} \rightarrow S\left(\left(S\left(\left(R^{1}\right)_{2}\right) \rightarrow x\right)_{2}\right)\right) \\
& =R^{2} f\left(S\left(\left(R^{1}\right)_{3}\right) \rightarrow x_{1}\right)\left(\left(R^{1}\right)_{1} S\left(\left(R^{1}\right)_{2}\right) \rightarrow S\left(x_{2}\right)\right) \\
& =R^{2} f\left(S\left(\left(R^{1}\right)_{2}\right) \rightarrow x_{1}\right)\left(\varepsilon_{t}\left(\left(R^{1}\right)_{1}\right) \rightarrow S\left(x_{2}\right)\right) \\
& =R^{2} f\left(S\left(R^{1}\right)_{L}\left(1_{(2)}\right) \rightarrow x_{1}\right)\left(S_{L}\left(1_{(1)}\right) \rightarrow S\left(x_{2}\right)\right) \\
& =R^{2}\left(R^{1} \rightarrow f\right)_{\left(x_{1}\right) S\left(x_{2}\right)} \\
& =R^{2} \otimes \theta_{H}\left(\left(R^{1} \rightarrow f\right)_{0} \otimes\left(R^{1} \rightarrow f\right)_{1}\right)(x) .
\end{aligned}
$$


It implies that $R^{2} \otimes\left(R^{1} \rightarrow f\right)_{0} \otimes\left(R^{1} \rightarrow f\right)_{1}=R^{2} r^{2} \otimes R^{1} \rightarrow f_{0} \otimes r^{1} \rightarrow f_{1}, f \in H^{*}$. Using the equality $l \rightarrow f h=\left(l_{1} \rightarrow f\right)\left(l_{2} \rightarrow h\right)$ we compute

$$
\begin{aligned}
f^{-1} h^{-1} \otimes f^{0} h^{0} & =R^{2} r^{2} \otimes\left(R^{1} \rightarrow f\right)\left(r^{1} \rightarrow h\right) \\
& =R^{2} \otimes\left(R^{1} \rightarrow f h\right) \\
& =(f h)^{-1} \otimes(f h)^{0} \\
& =\sigma(f h) .
\end{aligned}
$$

Finally we show that $\rho_{H^{*}}(l \rightarrow f)=l_{1} \rightarrow f_{0} \otimes l_{2} \rightarrow f_{1}$. Since for $l \in L, f \in H^{*}, x \in H$

$$
\begin{aligned}
& \theta_{H}\left(\left(l_{1} \rightarrow f_{0}\right) \otimes\left(l_{2} \rightarrow f_{1}\right)\right)(x) \\
& =\left(l_{1} \rightarrow f_{0}\right)\left(R^{2} \rightarrow x\right)\left(R^{1} l_{2} \rightarrow f_{1}\right) \\
& =f_{0}\left(S_{L}\left(l_{1}\right) R^{2} \rightarrow x\right)\left(R^{1} l_{2} \rightarrow f_{1}\right) \\
& =f_{0}\left(R^{2} S_{L}\left(l_{2}\right) \rightarrow x\right)\left(l_{1} R^{1} \rightarrow f_{1}\right) \\
& =f_{0}\left(R^{2} \rightarrow\left(S_{L}\left(l_{2}\right) \rightarrow x\right)\right)\left(l_{1} \rightarrow\left(R^{1} \rightarrow f_{1}\right)\right) \\
& =f\left(\left(S_{L}\left(l_{3}\right) \rightarrow x\right)_{1}\right)\left(l_{1} \rightarrow S\left(\left(S_{L}\left(l_{2}\right) \rightarrow x\right)_{2}\right)\right) \\
& =f\left(S_{L}\left(l_{3}\right) \rightarrow x_{1}\right)\left(l_{1} \rightarrow S\left(S_{L}\left(l_{2}\right) \rightarrow x_{2}\right)\right) \\
& =f\left(S_{L}\left(l_{3}\right) \rightarrow x_{1}\right)\left(l_{1} S_{L}\left(l_{2}\right) \rightarrow S\left(x_{2}\right)\right) \\
& =f\left(S_{L}\left(l_{2}\right) \rightarrow x_{1}\right)\left(\varepsilon_{t}\left(l_{1}\right) \rightarrow S\left(x_{2}\right)\right) \\
& =f\left(S_{L}\left(1_{(2)} l\right) \rightarrow x_{1}\right)\left(S_{L}\left(1_{(1)}\right) \rightarrow S\left(x_{2}\right)\right) \\
& =f\left(S_{L}(l) \rightarrow\left(1_{(1)} \rightarrow x_{1}\right)\right) S\left(1_{(2)} \rightarrow x_{2}\right) \\
& =f\left(S_{L}(l) \rightarrow x_{1}\right) S\left(x_{2}\right)=(l \rightarrow f)\left(x_{1}\right) S\left(x_{2}\right) \\
& =\theta_{H}\left((l \rightarrow f)_{0} \otimes(l \rightarrow f)_{1}\right)(x) .
\end{aligned}
$$

From all above, $H^{*}$ is a right $H$-Hopf module in ${ }_{L}^{L} \mathscr{O}$.

Applying Theorem 4.2 we can obtain the following result.

Corollary 4.3. $H^{*}$ is defined a right $H$-Hopf module in ${ }_{L}^{L} \mathscr{Y O}$ as above, then $H^{*}{ }^{* O H} \otimes_{t} H \cong H^{*}$.

\section{Applications}

As a consequence the space of coinvariants of the finite dimensional Hopf algebra is free of rank one. This is the case for the weak Hopf algebra in the category of the Yetter-Drinfeld modules.

Theorem 5.1. If $H$ is a finite-dimensional weak Hopf algebra in ${ }_{L}^{L} \mathscr{Y O}$. Then

1) $\operatorname{dim} H^{*} \mathrm{COH}=1$. Fix $\phi \neq 0 \in H^{*} \mathrm{COH}$.

2) The map $\theta: H \rightarrow H^{*}, \phi \otimes h \rightarrow \phi h$ is an right $H$-module and an right $H$-comodules isomorphism. In particular $H$ is a Frobenius weak Hopf algebra with Frobenius map $\phi$.

3) There exist a right integral $t$ in $H, \quad \chi_{L} \in A \operatorname{Ag}(L, k)$ and a group-like elment $g_{L}$ in $L$ such that for all $x \in H \quad \phi(t x)=\varepsilon_{H}(x)$

a) $\phi(t x)=\varepsilon_{H}(x)$,

b) $\phi(l \rightarrow x)=\chi_{L}(l) \phi(x)$,

c) $\phi(x) g_{L}=\phi\left(R^{1} \rightarrow x\right) S_{L}^{-1}\left(R^{2}\right)$

d) $l \rightarrow t=\chi_{L}(l) t$, for all $l \in L$.

4) The map $\theta: H \rightarrow H^{*}$ is a left $L$-semilinear and a left $L$-semicolinear in the sense that for all $h \in H$ 
$\theta(l \rightarrow h)=\chi_{L}\left(l_{1}\right) l_{2} \rightarrow \theta(h), \quad \sigma(\theta(h))=\theta(h)^{-1} \otimes \theta(h)^{0}=g_{L} R^{2} \otimes \theta\left(R^{1} \rightarrow h\right)$.

Proof. 1) Since $H^{* \mathrm{COH}}$ is a right $H$-Hopf module in ${ }_{L}^{L} / /$, we have $H^{* \mathrm{COH}} \otimes H \cong H^{*}, f \otimes h \rightarrow f h$. Since $\operatorname{dim} H^{*}=\operatorname{dim} H$, it follows that $\operatorname{dim} H^{*}=1$.

2) Choose $\phi \neq 0 \in H^{*} \mathrm{CoH}$. Then by (1) $\theta: H \rightarrow H^{*}, \phi \otimes h \rightarrow \phi h$ is an right $H$-modules and an right $H$-comodules. Thus $H$ is Frobenius weak Hopf algebra.

3) a) Since $H \cong H^{*}(\phi \otimes h \rightarrow \phi h)$, there is a unique element $t$ in $H$ such that $\phi t=\varepsilon_{H}$, i.e. $\phi(t x)=\varepsilon_{H}(x)$. For all $h \in H$ we have $(\phi t h)(x)=(\phi t)(h x)=\varepsilon_{H}(h x)=\varepsilon_{H}\left(\varepsilon_{s}(h) x\right)=\left(\phi t \varepsilon_{s}(h)\right)(x)$. It follows that th $=t \varepsilon_{s}(h), h \in H$. So $t$ is a right integral in $H$.

b) We remark that $S_{L}^{-1}(l) \rightarrow \phi \in\left(H^{*}\right)^{\mathrm{CoH}}$ for all $l \in L$ from Theorem 3.3. This implies $S_{L}^{-1} \rightarrow \phi=\chi_{L}(l) \phi$, i.e. $\phi(l \rightarrow x)=\chi_{L}(l) \phi(x), x \in H$ for some $\chi_{L}(l) \in \operatorname{Alg}(L, k)$, by $\operatorname{dim} H^{*}{ }^{*} O H=1$.

c) From Theorem 3.3 we have $H^{*} \mathrm{COH}$ is a right $L$-comodule, i.e. $\sigma(\phi) \in L \otimes\left(H^{*}\right)^{\mathrm{COH}}$. By $\operatorname{dim} H^{{ }^{*} \mathrm{CoH}}=1$ we can obtain $\sigma(\phi)=g_{L} \otimes \phi$ for some group-like element $g_{L}$ in $L$. This implies that $\phi(x) g_{L}=\phi\left(R^{1} \rightarrow x\right) S^{-1}\left(R^{2}\right)$.

d) Applying $\phi(l \rightarrow x)=\chi_{L}(l) \phi(x)$ we have

$$
\begin{aligned}
\phi(l \rightarrow t) x & =\phi\left(\left(l_{1} \rightarrow t\right)\left(l_{2} S_{L}\left(l_{3}\right) \rightarrow x\right)\right),=\phi\left(l_{1} \rightarrow\left(t\left(S_{L}\left(l_{2}\right) \rightarrow x\right)\right)\right) \\
& =\chi_{L}\left(l_{1}\right) \varepsilon\left(S_{L}\left(l_{2}\right) \rightarrow x\right)=\chi_{L}\left(1_{(1)} l\right) \varepsilon\left(S_{L}\left(1_{(2)}\right) \rightarrow x\right) \\
& =\chi_{L}(l) \chi_{L}\left(1_{(1)}\right) \varepsilon\left(S_{L}\left(1_{(2)}\right) \rightarrow x\right) \\
& =\chi_{L}(l) \phi\left(1_{(1)} \rightarrow t\left(S_{L}\left(1_{(2)}\right) \rightarrow x\right)\right) \\
& =\chi_{L}(l) \phi\left(\left(1_{(1)} \rightarrow t\right)\left(1_{(2)} S_{L}\left(1_{(3)}\right) \rightarrow x\right)\right) \\
& =\chi_{L}(l) \phi\left(\left(1_{(1)} \rightarrow t\right)\left(1_{(2)} \rightarrow x\right)\right) \\
& =\chi_{L}(l) \phi(t x)=\left(\phi \chi_{L}(l) t\right)(x),
\end{aligned}
$$

This means $l \rightarrow t=\chi_{L}(l) t$, for all $l \in L$.

4) For all $x \in H$ we have

$$
\begin{aligned}
\theta(l \rightarrow h)(x) & =\phi((l \rightarrow h) x)=\phi\left(l_{1} \rightarrow\left(h\left(S_{L}\left(l_{2}\right) \rightarrow x\right)\right)\right), \\
& =\chi_{L}\left(l_{1}\right) \phi\left(h\left(S_{L}\left(l_{2}\right) \rightarrow x\right)\right)=\chi_{L}\left(l_{1}\right) \theta(h)\left(S_{L}\left(l_{2}\right) \rightarrow x\right), \\
& =\chi_{L}\left(l_{1}\right)\left(l_{2} \rightarrow \theta(h)(x)\right)=\left(\chi_{L}\left(l_{1}\right) l_{2} \rightarrow \theta(h)\right)(x) .
\end{aligned}
$$

This implies $\theta(l \rightarrow h)=\chi_{L}\left(l_{1}\right) l_{2} \rightarrow \theta(h)$.

$$
\sigma(\theta(h))=(\phi h)^{-1} \otimes(\phi h)^{0}=g_{L} h^{-1} \otimes \phi h^{0}=g_{L} R^{2} \otimes \theta\left(R^{1} \rightarrow h\right)
$$

\section{Acknowledgements}

The author would like to thank the referee for many suggestions and comments, which have improved the overall presentations.

\section{Funding}

Research supported by the Project of Shandong Province Higher Educational Science and Technology Program (J12LI07) and the Project of National Natural Science Foundation of China (51078225).

\section{References}

[1] Böhm, G., Nill, F. and Szlachányi, K. (1999) Weak Hopf Algebras I. Integral Theory and C*-Structure. Journal of Al- 
gebra, 221, 385-438. http://dx.doi.org/10.1006/jabr.1999.7984

[2] Caenpeel, S, Wang, D.-D. and Yin, Y.-M. (2005) Yetter-Drinfeld Modules over Weak Bialgebras. Annali Dell'universitia DI Ferrarà, Sezione VII-Scienze Matematiche, 51, 69-98.

[3] Kadison, L. and Nikshych, D. (2001) Frobenius Extensions and Weak Hopf Algebras. Journal of Algebra, 244, 312342. http://dx.doi.org/10.1006/jabr.2001.8911

[4] Takeuchi, M. (1998) Hopf Modules in Yetter-Drinfeld Categories. Communications in Algebra, 26, 3057-3070. http://dx.doi.org/10.1080/00927879808826327

[5] Bohm, G. (2000) Doi-Hopf Modules over Weak Hopf Algebras. Communications in Algebra, 28, 4687-4689.

[6] Shen, B.-L. and Wang, S.-H. (2009) Weak Hopf Algebra Duality in Weak Yetter-Drinfeld Categories and Applications. Internation Electeronic Journal of Algebra, 6, 74-94.

[7] Yin, Y.-M. and Zhang, M.-C. (2011) Hopf Modules in the Braided Monoidal Category ${ }_{L}$ M. Le Matematiche, LXVI, 81-92. 